\title{
Application Article \\ Performance Comparison of Reflector- and Planar-Antenna Based Digital Beam-Forming SAR
}

\author{
Marwan Younis, Sigurd Huber, Anton Patyuchenko, Federica Bordoni, \\ and Gerhard Krieger
}

Microwaves and Radar Institute, German Aerospace Center (DLR), 82230 Wessling, Germany

Correspondence should be addressed to Marwan Younis, marwan.younis@dlr.de

Received 15 October 2008; Revised 23 January 2009; Accepted 17 March 2009

Recommended by Giovanni Toso

The trend in the conception of future spaceborne radar remote sensing is clearly toward the use of digital beamforming techniques. These systems will comprise multiple digital channels, where the analog-to-digital converter is moved closer to the antenna. This dispenses the need for analog beam steering and by this the used of transmit/receive modules for phase and amplitude control. Digital beam-forming will enable Synthetic Aperture Radar (SAR) which overcomes the coverage and resolution limitations applicable to state-of-the-art systems. On the other hand, new antenna architectures, such as reflectors, already implemented in communication satellites, are being considered for SAR applications. An open question is the benefit of combining digital beamforming techniques with reflector antennas. The paper answers this question by comparing the system architecture and digital beam-forming requirements of a planar and a reflector antenna SAR. Further elaboration yields the resulting SAR performance of both systems. This paper considers multiple novel aspects of digital beam-forming SAR system design, which jointly flow into the presented system performance.

Copyright (C) 2009 Marwan Younis et al. This is an open access article distributed under the Creative Commons Attribution License, which permits unrestricted use, distribution, and reproduction in any medium, provided the original work is properly cited.

\section{Introduction}

Synthetic aperture radar (SAR), utilizing digital beamforming, is increasingly being considered for future missions. This is evident both from research activities $[1,2]$ and space qualified technology demonstrations [3]. One of the reasons for this trend is that state-of-the-art SAR systems cannot fulfill he heterogeneous demand on products at the required performance level. The motivation for using Digital Beam-Forming (DBF) techniques is their ability to provide a simultaneous wide swath (coverage) and high resolution. In this paper, systems utilizing the various forms of DBF, such as SCan-On-REceive (SCORE), multiple azimuth receive channels, and transmit phase center variations are jointly referred to as SMART, which stands for Smart Multi-aperture Radar Techniques. In the most general sense SMART sensors allow a relaxation of the system design parameters, by increasing the degrees of freedom. Specifically, for a given geometric resolution this results in systems with higher Signal-to-Noise Ratio (SNR) and lower ambiguity-to-signal ratio, both being key requirements on SAR systems. Equipped with digital receive channels, SMART sensors do not require phase and amplitude control of the received signals (analog beam-forming); this yields an RF hardware free of transmit/receive modules and complex control and calibration units. Instead, SMART will push the development of onboard digital signal processing capable of handling multiple channels of high data rate.

This paper discusses the use of SMART in conjunction with two different antenna concepts. On one side a planar array, consisting of a single small transmit antenna and a receive antenna array with multiple subapertures in elevation is considered. The array is equipped with digital receive channels and onboard DBF is performed on the data. On the other side we consider a reflector antenna with a feed consisting of multiple transmit/receive elements in elevation. Here beam-forming is performed both on transmit and on receive by selecting the active elements of the array.

The paper analyzes and compares the system concepts in terms of realization complexity, space suitability, hardware issues, digital processing requirements and, last but not least, 
the resulting SAR performance. The focus of the paper is on different antenna issues, rather than the SMART SAR operational modes which are detailed for example in [1].

The paper starts by introducing digital beam-forming in elevation and azimuth in Section 2. Next the system architecture and signal processing approach for each of the planar and reflector system are described in Section 3. The parameters used to characterize the SAR performance are defined in Section 4, which includes several new performance criteria necessary for DBF systems. Then, in Section 5, the performance requirements are stated and the design parameters common to both systems are given. This is followed by the specific design and SAR performance analysis of each system in Sections 6 and 7. Finally Section 8 summarizes the paper and gives an outlook to future prospects.

\section{System Operation}

2.1. Digital Beam-Forming in Elevation. In 1981 Blythe [4] suggested a basic approach for analog beam-steering such that the receive beam moves over the swath in accordance with the direction of reflection. About twenty years later, his idea finds a more detailed description and justification in the independent and almost contemporary works by Kare [5], and Suess and Wiesbeck [6].

The patent by Kare in [5] presents the Moving Receive Beam as a technique to improve the quality of "highresolution SAR imagery over a wide target area," by reducing the edge losses and the range ambiguities. Different approaches for the steering of the receiving beam are suggested: based on an a priori assumption on the acquisition geometry, open-loop system on the actual receive data, closeloop system or on test pulses. Moreover, Kare describes different analog feed implementation options, involving both planar array and reflector architectures, showing a thorough understanding of the implications in the reflector case. The necessity to take into account the temporal extension of the pulse in the steering mechanism is mentioned, and in particular for chirp pulses, the possibility to adapt the position of the beam to the frequency of the received signal.

In $[6,7]$ for the first time digital beam-forming techniques are presented in conjunction with a time varying receive beam-steering in elevation. A quantitative description of the steering law, comprehensive of a compensation for the time spread of chirp pulses by using a frequency dependent steering is given.

The SCan-On-Receive (SCORE) mode of operation is primarily based on generating a wide transmit beam that illuminates the complete swath and a narrow, high-gain beam on receive that follows the pulse echo on the ground. The high-gain SCORE beam results in an increased signal-tonoise ratio compensating the low-gain (wide beam) transmit antenna loss. Specifically at the swath edges (half-power beamwidth angles) the typical two-way loss of a monostatic system is reduced. Further, the narrow receive beam has the advantage of attenuating the range ambiguities. For a strictly spherical Earth model, that is, no topographic height, the direction of arrival of the received echo is a function of the slant range. This results in a one-to-one relation between the required beam steering angle and the time variable. DBF is used to combine the signals received by the subapertures, in order to obtain at each instant a sharp and high-gain pattern, steered toward the expected direction of arrival of the echo. The specific beam-forming implementation depends on the system involved, but can in general be described by a complex and time varying weighting of the subaperture followed by a summation. This effectively reduces the data rate by eliminating the redundancies; thus, in the ideal case the data reduction is lossless. ( This reduction of data rate is the case for DBF in elevation, where the channels are combined. In general, however, DBF results in a higher data rate since it facilitates a higher resolution and wider coverage, that is, higher information rate.)

2.2. Digital Beam-Forming in Azimuth. Multiple phase centers in along-track (azimuth) enable an improved azimuth resolution, while requiring the data streams from each azimuth channel to be recorded separately for on-ground processing [8]. Here, in contrast to the elevation operation, the multiple channels yield in a higher data volume resulting from the increased resolution. The principle behind multiazimuth channel operation is different for the planar and reflector systems, which requires a separate treatment.

For the planar system, shown in Figure 1(a), all subapertures cover the same angular segment, thus "seeing" identical Doppler spectra. Considering a single subaperture, the spacial separation between the samples, as determined by the pulse repetition frequency $(P R F)$, is such that the Doppler spectrum is undersampled, that is, aliased or ambiguous. It is only through the combination of the spacial samples of all subapertures, that the Doppler spectrum can be recovered unambiguously. Each subaperture can thus be considered as an additional spacial sample in the along-track direction carrying nonredundant information. In general the system operation will be such that the PRF will result in a nonuniform spacial sampling; this makes the combination of the channels a nontrivial task, requiring a reconstruction of the Doppler spectrum which is detailed in [9] based on a generalization of the sampling theorem $[10,11]$.

A reflector system of multiple azimuth phase channels will require multiple feeds displaced in along track direction as shown in Figure 1(b). In contrast to the planar case, here each azimuth element "looks" at a different angle and by this the angular segment (Doppler span) covered by each element does not overlap with those of the others. Thus each element samples a narrow Doppler spectrum corresponding to the half-power-beamwidth of the corresponding pattern. The PRF must be high enough such that the spacial sampling for each channel is adequate. If the Doppler spectra of the elements are contiguous, they jointly yield a higher azimuth resolution [2]. Here also, each channel carries nonredundant information.

The above description pointed out the multiazimuth channel operation for improved resolution. However, the systems detailed in this paper do not utilize multiple azimuth channels, since this is not the issue of this paper. 


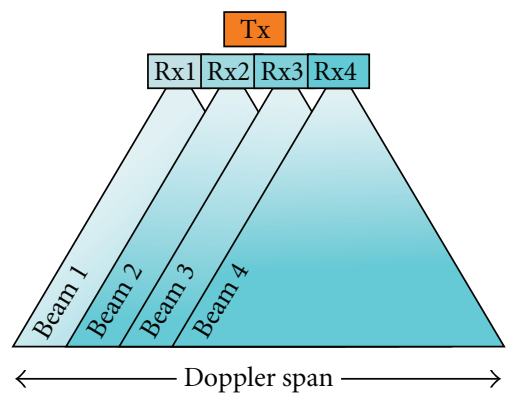

(a) Planar system

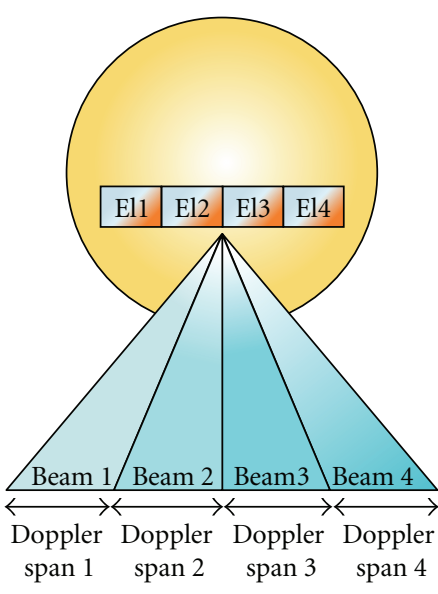

(b) Reflector system

Figure 1: MultiAzimuth channels yield identical wide Doppler span for each channels in case of the planar system (a), and narrow Doppler spans of different Doppler centroid in the case of the reflector system (b).

\section{Basic Architecture}

Although the beams generated by the two systems are similar to each other, the underlying system architecture, used to generate these beams, is quite different. Therefore in the following the system architecture of each system is described.

3.1. Planar System. The planar receive array consists of $N_{\mathrm{pl}}$ subapertures, uniformly placed in the plane perpendicular to the flight direction, with DBF capabilities in elevation, as shown in Figure 2. In spaceborne SAR, patch elements or slotted waveguide antennas are commonly used. For the basic operation mode considered in this paper, no beamforming capabilities are required on transmit and thus a single separate transmit antenna is used. The pattern of the transmit antenna coincides with that of each receive subaperture, by this each subaperture "sees" the same area on the ground. The echoes from the ground are received by each subaperture, amplified, downconverted and digitized. (Depending on the carrier frequency future systems may use direct digitization without a dedicated down conversion.) From this, it is evident that this system will require the capability to handle $N_{\mathrm{pl}}$ digital data streams, furthermore onboard DBF capability is required.

The data stream $\underline{s}_{i}(t)$ of each channel $i$ is multiplied by a time varying complex coefficient $\underline{w}_{i}(t)$. Then the data values are summed up resulting in the output signal, given in (1), which is saved onboard the instrument for later downlink.

$$
\underline{s}_{\mathrm{pl}}(t)=\sum_{i=1}^{N_{\mathrm{pl}}} \underline{w}_{i}(t) \cdot \underline{s}_{i}(t) .
$$

The complex multiplication and summation is equivalent to forming a narrow, time varying beam, which follows the echo on the ground. The beam will scan the complete swath within the time period of one pulse repetition interval $(\mathrm{PRI}=1 / \mathrm{PRF})$. Any imprecision in determining the complex weights will lead to a beam which is not steered in the signal's

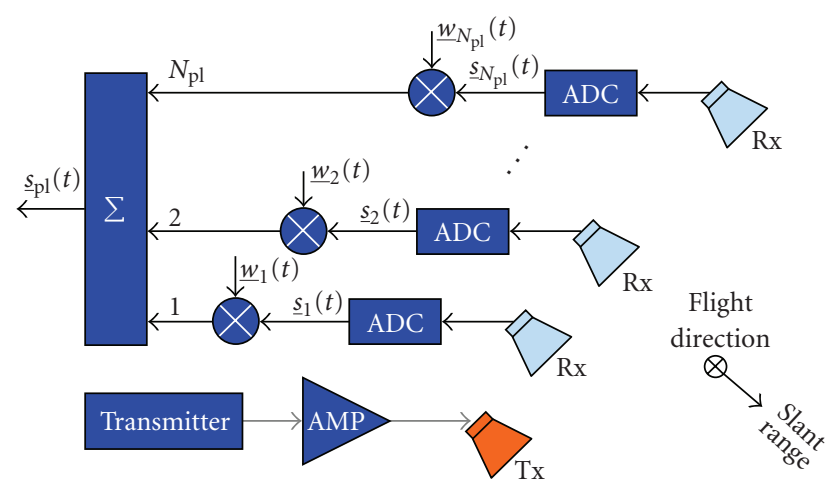

Figure 2: General system architecture for planar system; some components such as LNAs, mixers, filters etc. are not shown to maintain a clear representation.

direction-of-arrival and consequently to a loss in power. The generation of frequency dispersive beams (cf. later discussion on pulse extension loss in Section 4) as suggested in [6, 7] is also possible by introducing an additional time shift and adapting the weights; this can still be implemented in the time domain, that is, without a significant increase in processing complexity.

3.2. Reflector System. The reflector system consists of a parabolic reflector and a feed array of transmit/receive elements, as shown in Figure 3. The feed elements are arranged in the plane perpendicular to the flight direction and facing the reflector. Each element results in a beam, illuminating a region on the ground, which partially overlaps with the region illuminated by the beams of the adjacent elements. To illuminate a given angular segment in elevation, the corresponding feed elements are activated. Depending on the scan angle, one or more elements need to be activated, to avoid SNR loss (cf. Section 7). Similar to the planar system, the receive beam will scan the complete swath within the time period of one PRI, however here each element is only 


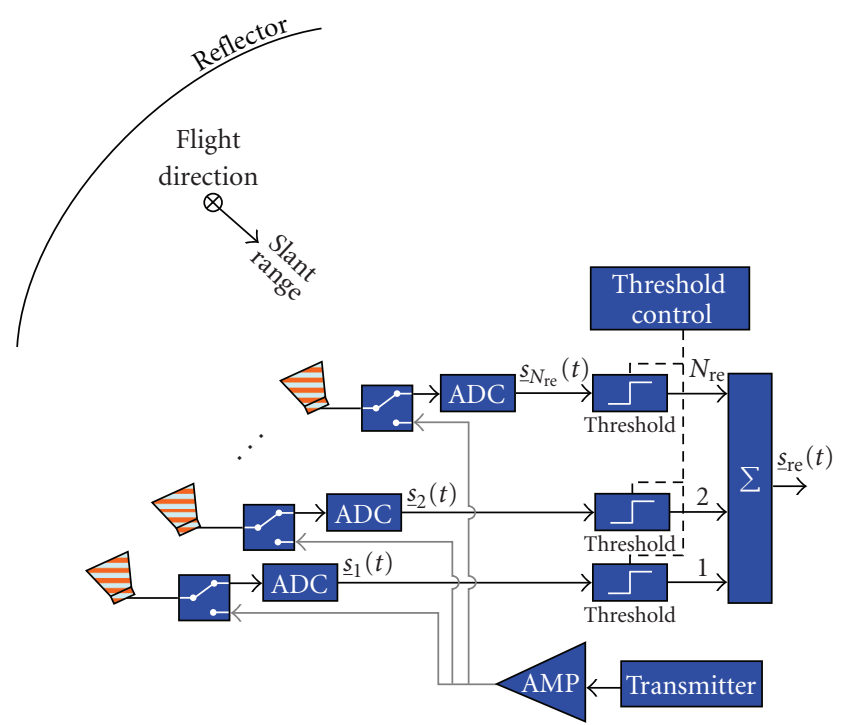

Figure 3: System architecture for reflector system; some components such as LNAs, mixers, filters etc. are not shown to maintain a clear representation.

active during a subinterval of this time period. On transmit all $N_{\text {re }}$ elements are activated generating a wide beam. (Note the difference to the planar case where a single subaperture generates a wide beam).

In the case of the reflector system, DBF consists of selecting a subset of the feed elements and summing up the corresponding data streams. For the given number of adjacent active elements, $N_{\text {act }}$, the output signal is given by:

$$
\underline{s}_{\mathrm{re}}(t)=\sum_{i=n}^{n+N_{\mathrm{act}}} \underline{s}_{i}(t), \quad 1 \leq n, N_{\mathrm{act}} \leq N_{\mathrm{re}} .
$$

Clearly the data handling complexity is below that of the planar system. An attractive realization of the subset selection is to use digital threshold detectors, to determine whether a data stream is passed to the summation or nulled. The argumentation here is that an echo from a certain direction-of-arrival will only contribute to the signal level of a few feed elements, corresponding to this directionof-arrival. The threshold levels need to be adapted to the (slow) variation of the reflectivity for different scenes, which causes a variation of the average power level at the receiver. For the same reason, the threshold levels of the different channels cannot be determined independently from each other. Thus the threshold ensures that at each time instance only the information relevant channels are summed up. For the SAR processing it is important to record which signals are summed at each instance in order to be able to reconstruct the actual antenna pattern.

\section{SAR Performance Parameter Definition}

This section provides a definition of the quantities involved in the evaluation of the SAR system performance.
Range-Ambiguity-to-Signal Ratio (RASR) quantifies the disturbance due to echoes from preceding and succeeding pulses, which arrive simultaneously with the echo of interest. The RASR is defined as the ratio between the total power received from these ambiguous signals and the power of the useful signal. The definition of the RASR as a function of slant range $R$ is given by [12]

$$
\operatorname{RASR}(R)=\frac{R^{3} \cdot \sin \eta}{\left|C_{2 \text { way,el }}(\vartheta)\right|^{2}} \sum_{\substack{l \neq 0 \\ l=-N_{N}}}^{N_{F}} \frac{\left|C_{2 \text { way,el }}\left(\vartheta_{l}\right)\right|^{2}}{R_{l}^{3} \cdot \sin \eta_{l}},
$$

where $C_{2 \text { way,el }}(\vartheta)=C_{T, \mathrm{el}}(\vartheta) \cdot C_{R, \mathrm{el}}(\vartheta)$ represents the twoway antenna pattern in elevation; $\vartheta$ is the beam steering angle; $\eta$ the local incidence angle corresponding to the signal; and $N_{N}, N_{F}$ the number of ambiguities considered in the calculation in near and far range, respectively. (Note that in the case of SCORE the transmit $C_{T, \mathrm{el}}\left(\vartheta_{l}\right)$ and receive $C_{R, \mathrm{el}}\left(\vartheta_{l}\right)$ elevation patterns are substantially different from each other (cf. Section 2.1)). The subscript $l$ denotes the corresponding quantities associated to the ambiguous signals. In the above expression the variation of the surface reflectivity with incidence angle is only considered through the $\sin \eta$ term, representing the geometrical projection of the surface area on the normal to the line-of-sight vector; otherwise the expression would include the radar backscatter coefficient (RCS).

Azimuth-Ambiguity-to-Signal Ratio (AASR) quantifies the aliasing generated by the finite sampling of the Doppler spectrum at intervals of the pulse repetition frequency $(P R F)$. In fact, the Doppler spectrum is not strictly band limited due to the sidelobes of the azimuth antenna pattern. As a consequence, Doppler frequency components outside the sampling interval $-\mathrm{PRF} / 2 \leq f_{D}<+P R F / 2$ are folded back into the processed Doppler frequency range, producing ambiguities. The AASR is defined as [12]

$$
\mathrm{AASR}=\frac{\sum_{m \neq 0, m=-N_{A}}^{N_{A}} \int_{-B_{p} / 2}^{B_{p} / 2}\left|C_{2 \text { way,az }}\left(f_{D}+m \cdot \mathrm{PRF}\right)\right|^{2} d f}{\int_{-B_{p} / 2}^{B_{p} / 2}\left|C_{2 \text { way }, \mathrm{az}}\left(f_{D}\right)\right|^{2} d f}
$$

where $f_{D}$ is the Doppler frequency; $B_{p}$ is the processed azimuth bandwidth; $C_{2 \text { way,az }}\left(f_{D}\right)$ the two-way (transmit/receive) azimuth antenna pattern as a function of the Doppler frequency; $N_{A}$ the number of ambiguities considered in the calculation. Note that there is a one-toone correspondence between the the Doppler frequency and the azimuth angle, $\phi$, given by $f_{D}=2 V \sin \phi / \lambda$, where $V$ is the platform velocity. This allows to express the azimuth pattern either through the Doppler frequency or through the azimuth angle. Further, the processed Doppler bandwidth, $B_{p}$, is fixed by the required azimuth resolution, $\delta_{\mathrm{az}}$, through $B_{p} \approx V / \delta_{\mathrm{az}}$.

Noise-Equivalent Sigma Zero (NESZ) is a measure of the sensitivity of the system to areas of low radar backscatter. It is given by the value of the backscatter coefficient 


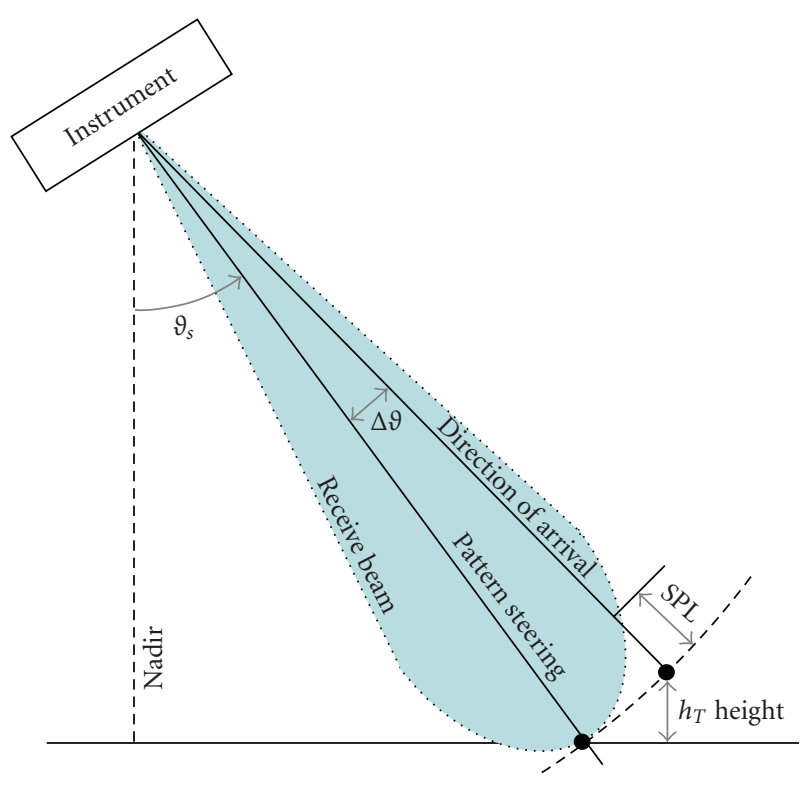

Figure 4: SCORE pattern loss SPL.

corresponding to a signal-to-noise ratio of unity, $\mathrm{SNR}=1$. The NESZ as a function of slant range $R$ is given by [13]

$$
\begin{aligned}
\operatorname{NESZ}(R)= & \frac{2(4 \pi)^{3} P_{n} \cdot \operatorname{PRF}}{c_{0} \lambda^{2} \cdot P_{\mathrm{av}} \cdot G_{T} G_{R}} \frac{N_{\mathrm{az}} \sin (\eta)}{\delta_{\mathrm{az}}} \\
& \cdot \frac{1}{\left|C_{2 \text { way,el }}(\vartheta) \cdot \sum_{i=1}^{N_{\mathrm{az}}}\left(C_{2 \text { way,az }}\left(\phi_{i}\right) / R^{2}\left(\phi_{i}, \vartheta\right)\right)\right|^{2}},
\end{aligned}
$$

where $P_{n}$ represents the equivalent receiver noise power; $P_{\mathrm{av}}$ is the average transmit power; $G_{T}, G_{R}$ are the antenna gain in transmission and reception, respectively; $\lambda$ is the radar wavelength; $c_{0}$ the speed of light; $\delta_{\mathrm{az}}$ is the azimuth resolution; $N_{\mathrm{az}}=\lambda R \cdot \mathrm{PRF} / 2 \delta_{\mathrm{az}} \cdot V$ is the number of integrated pulses during the azimuth compression.

SCORE Pattern Loss (SPL) is a measure of the antenna gain loss due to topographic height such as mountains and relief. In fact, the SCORE steering direction is computed based on an Earth model, that is, not including topographic height. As a consequence, in a realistic scenario characterized by mountains and relief, there is a mismatch between the actual direction of arrival of the signal echo and the steering direction. This results in a gain loss with respect to the ideal operational condition.

As shown in Figure 4 the SPL results because of the angle difference, $\Delta \vartheta$, between the steering direction, $\vartheta_{s}$ (the maximum of the pattern), and the echo actual direction of arrival. Since the antenna steering angle is computed using the correspondence between the signal delay and angle of arrival, which is in general based on a simplified geometrical model of the Earth, any steering error will also result in a SCORE pattern loss. For each instant of time the SPL is defined by the value of the elevation receive pattern at

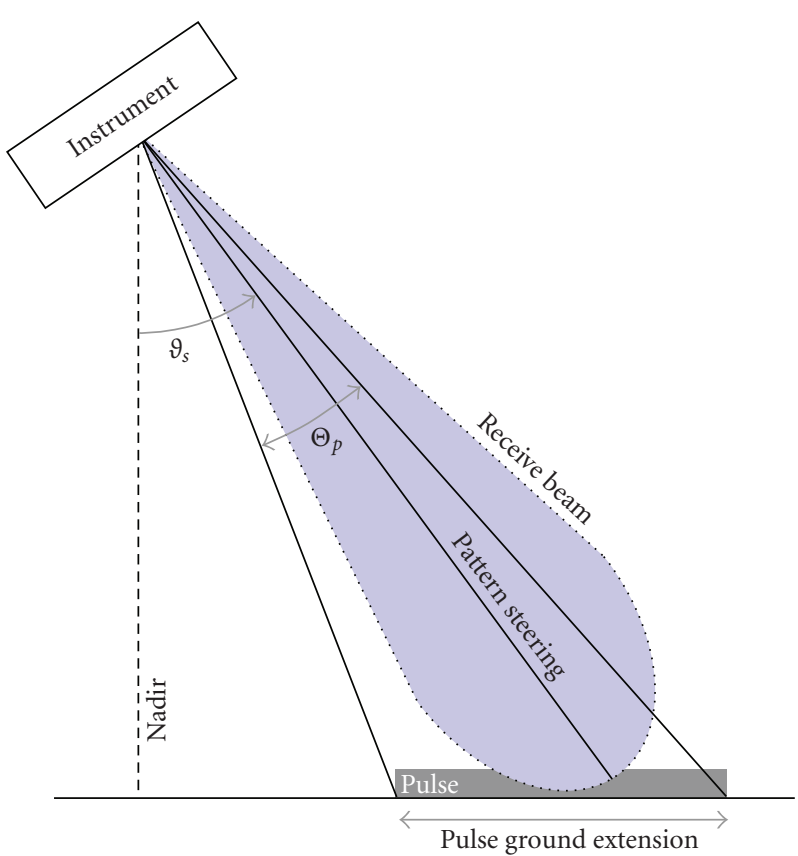

Figure 5: Pulse extension loss PEL.

the angle $\vartheta_{s}+\Delta \vartheta$ normalized to the maximum value of the pattern at that time:

$$
\mathrm{SPL}=\frac{C_{R, \mathrm{el}}\left(\vartheta_{s}+\Delta \vartheta\right)}{C_{R, \mathrm{el}}\left(\vartheta_{s}\right)}
$$

Note that in the context of the above definition negative $S P L$ values indicate a loss in gain. (The transmit pattern does not appear in the expression since it does not affect the SPL).

Pulse Extension Loss (PEL) is the loss associated to the non-vanishing pulse duration, as shown in Figure 5. In fact the backscattered echo is characterized by an instantaneous extension $c_{0} \tau_{p} / 2 \sin \eta$, on the ground simultaneously contributing to the received power at each instance of time. When the angular segment, $\Theta_{p}$, corresponding to this extension becomes comparable to, or larger than the receive beamwidth, a loss occurs. The PEL is defined as the ratio of $\Theta_{p}$ weighted by the receive pattern steered to the pulse center to $\Theta_{p}$ weighted by the maximum of the pattern (Due to the finite number of elevation patterns the maximum of the pattern may not be at the pulse center, however, the loss caused by this pulse switching is already accounted for in the computation of the NESZ, for which it is not included in the $P E L$.) Then, the PEL is computed according to

$$
\mathrm{PEL}=\frac{1}{C_{R, \mathrm{el}}\left(\vartheta_{s}\right) \cdot \Theta_{p}} \cdot \int_{\vartheta_{s}-\Theta_{p} / 2}^{\vartheta_{s}+\Theta_{p} / 2} C_{R, \mathrm{el}}(\vartheta) d \vartheta
$$

where the angular pulse ground extension $\Theta_{p}\left(\tau_{p}, \eta\right)$ is a function of the pulse duration $\tau_{p}$ and incidence angel $\eta$, and the integral limits are taken to be symmetrical with respect to the maximum of the pattern at $\vartheta_{s}$.

It is worth noting that the PEL is an integral loss including all point scatterers within the pulse extension at 


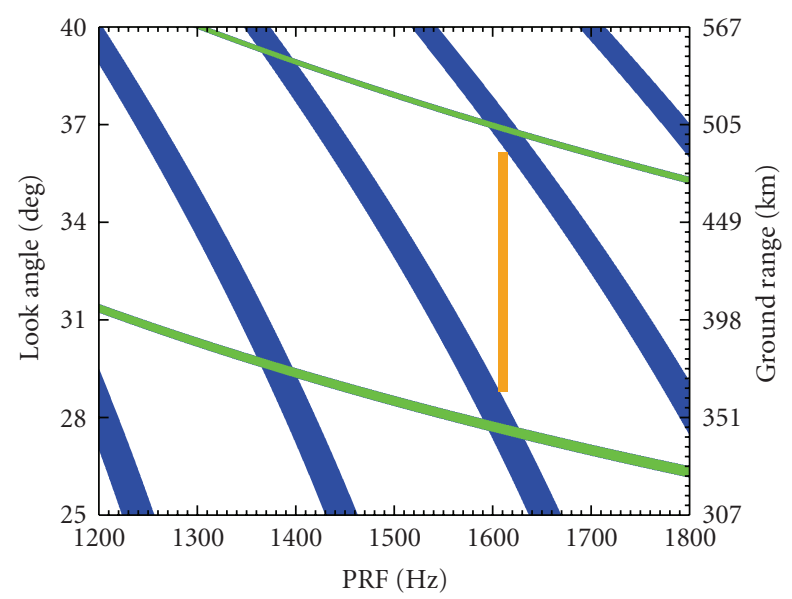

FIGURE 6: Timing (diamond) diagram for an orbit height of $650 \mathrm{~km}$ and a transmit duty cycle of $8 \%$. For a given $P R F$ the diagram shows the restrictions on the receive window due to transmit instances (wide dark strips) and nadir echos (thin light strips).

any instance of time. Thus, the PEL does not characterize the response of an individual point scatterer over time. In fact the received chirp signal from any single point scatterer will show an amplitude and phase modulation. This modulation can detoriate the impulse response in range for narrow receiver patterns or a large number of beam switchings.

\section{SAR Requirement and Common Design Parameters Definition}

5.1. Performance Requirements. Table 1 collects the main performance requirements on SAR image quality, which has been assumed to design the reference system. The reported values have been selected according to the most advanced or forthcoming SAR mission performance. It is worth noting that a standard on SAR image quality does not exist. For comparison, current satellite missions, such as COSMOSkyMed [14] and TerraSAR-X [15], deliver products with a coverage ranging from $10 \mathrm{~km}$ to $200 \mathrm{~km}$ and a corresponding resolution ranging from $1 \mathrm{~m}$ to $100 \mathrm{~m}$, respectively. The ambiguity and NESZ level are in the order of $-21 \mathrm{~dB}$ and $-24 \mathrm{~dB}$, respectively.

5.2. Common Design. In the following the system design parameters common to both systems are stated. This is the basis for the individual planar array and reflector antenna system designs in Sections 6 and 7, respectively, which are optimized to yield the best individual SAR performance.

The systems considered in this paper operate at C-band frequencies, however the presented findings are mostly also applicable to other frequency bands. Further, for simplicity, the investigation is for a single polarization system.

The orbit height selection has a major influence on the radar parameters, such as the incidence angle range to cover a given swath, the path attenuation and by this the $S N R$, as well as the resulting mission scenario such as repeat cycles. Here
TABLE 1: Requirement on performance parameters.

\begin{tabular}{|c|c|c|}
\hline Parameter & & Value \\
\hline Frequency & C-Band & $5.4 \mathrm{GHz}$ \\
\hline Swath width & & $\geq 120 \mathrm{~km}$ \\
\hline Global coverage & & Within one repeat cycle \\
\hline $\begin{array}{l}\text { Resolution (range, } \\
\text { azimuth) }\end{array}$ & $\delta_{r g}, \delta_{a z}$ & $8 \times 8 \mathrm{~m}$ \\
\hline $\begin{array}{l}\text { Ambiguity-to-signal } \\
\text { ratio }\end{array}$ & RASR, AASR & $-20 \mathrm{~dB}$ \\
\hline $\begin{array}{l}\text { Noise-equivalent sigma } \\
\text { zero }\end{array}$ & NESZ & $-25 \mathrm{~dB}$ \\
\hline SCORE loss @1.5km & $S P L$ & $\leq 1 \mathrm{~dB}$ \\
\hline
\end{tabular}

a $650 \mathrm{~km}$ orbit is selected. This together with a requirement of a $120 \mathrm{~km}$ swath will allow a global coverage of the Earth within a single repeat cycle of 23 days.

Next an investigation of the timing is due in order to determine the specific range of incidence angles and $P R F$ values. The timing diagram indicates whether the backscattered echo from a point on the ground can be received, that is, whether the echo delay is such that the signal does not superimpose neither on the transmit instances nor on the nadir echoes. The timing diagram is a function of the pulse repetition frequency and the angular position of the backscattering point within the swath. Figure 6 shows the timing diagram for a transmit pulse duty cycle of $8 \%$. Choosing PRF $=1610 \mathrm{~Hz}$ results in a receive window, which covers the look angle range $\left[28.8^{\circ}, 36.1^{\circ}\right]$, which corresponds to a maximum swath width of $125 \mathrm{~km}$.

For the simple stripmap mode of operation considered here, the look angle range directly gives the half power beamwidth in elevation for the transmit antenna, which is set to $\Theta_{T, \text { el }}=7.3^{\circ}$. The corresponding receive half power beamwidth is not a common design parameter, since its value will be different for the planar and reflector system (cf. Sections 6 and 7).

The half power beamwidth in azimuth is determined by the requirement on the azimuth resolution $\delta_{\mathrm{az}}$ and, to some extend, the required $A A S R$. For the systems considered here, the transmit and receive antennas have the same coverage in azimuth. For a given resolution, the half power beamwidth can be approximated by $\Theta_{\mathrm{az}} \approx \lambda / 2 \delta_{\mathrm{az}}$. This results in a half power beamwidth in the order of $0.2^{\circ}$.

A design parameter, which is crucial both for the SAR performance and the instrument design, is the transmit power. Here the average transmit power together with the hardware efficiency influences (1) the amount of heat dissipation and by this the thermal design of the instrument; (2) the size of the solar panel and the orbit usage of the instrument; (3) the NESZ of the SAR. On the other side, the maximum peak power determines the minimum duty cycle (for a given NESZ) which in turn influences the timing. Both systems analyzed in this paper have the same average transmit power of $P_{\mathrm{av}}=300 \mathrm{~W}$ and a duty cycle of $8 \%$. 


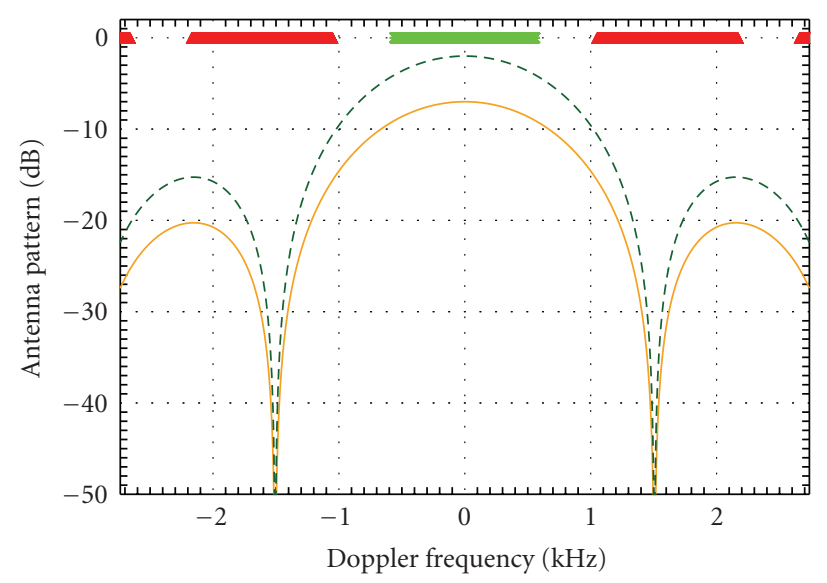

(a) Azimuth patterns

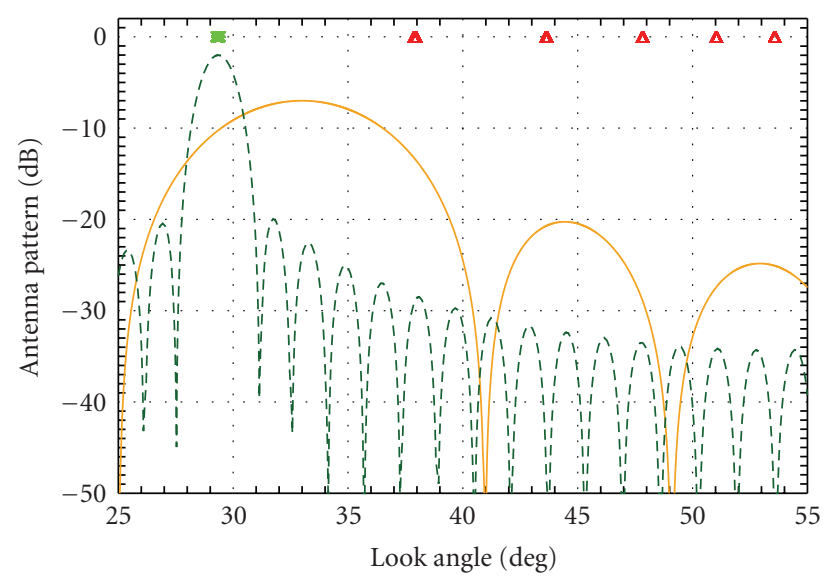

(b) Elevation patterns

Figure 7: Transmit (solid line) and receive patterns (dashed line) of the planar antenna system in azimuth and elevation. All patterns are normalized to the maximum gain of the uniformly tapered receive array.

\section{Design and Performance of Planar System}

In the following, first the design of the planar system is derived, which is mainly the dimensioning of the antenna, then the SAR performance of the system is given.

6.1. Planar System Design. In the case of the planar array antenna structure, the receive antenna is composed of 20 subapertures, placed in the elevation direction and only a single azimuth subaperture (channel) is employed. The dimensions of the antenna strongly affect the system performance; here the azimuth ambiguity requirement is the driving factor for the antenna length. In particular, the length of both transmit and receive antennas is set to $10 \mathrm{~m}$, which is affordable from a practical point of view in terms of cost and launcher requirement. To limit the azimuth ambiguities, the processed Doppler bandwidth is reduced at the cost of a worsened azimuth resolution. The azimuth patterns are shown in Figure 7(a), no amplitude taper is applied in azimuth, and transmit and receive patters are identical, however, the patterns are normalized to the maximum gain of a uniformly tapered receive array, which results in the difference in the level between the two azimuth patterns.

The elevation dimension of the transmit antenna is set in order to illuminate the swath of interest with the mainlobe pattern. For a uniform rectangular aperture illumination the half beamwidth is roughly given by the radar wavelength normalized by the antenna length $0.89 \cdot \lambda / d$. For the half power beamwidth of Section 5.2 this corresponds to an antenna height of $\approx 0.4 \mathrm{~m}$. The elevation patterns are shown in Figure 7(b) where an amplitude taper is applied to the receive array in order to reduce the side lobe level to $20 \mathrm{~dB}$. It is worth remarking that for a given average transmitted power, the NESZ performance mainly depends on the antenna area. Thus increasing the antenna height allows improving the radiometric resolution, however at the cost of an increased sensitivity of the system to surface topographic height and an increased pulse extension loss.
For the system under study, the receive antenna height is $2 \mathrm{~m}$ such that the half power beamwidth of the receive pattern is about $1.6^{\circ}$.

6.2. Planar System Performance. The ambiguity performance of the planar system is shown in Figure 8 versus the ground range position on the swath. The AASR is constant all over the swath, due to stripmap operational mode. Its value, which depends on the PRF, the antenna length, and the processed azimuth bandwidth is around $-22 \mathrm{~dB}$ for an azimuth resolution of $\delta_{\mathrm{az}}=6 \mathrm{~m}$.

The RASR varies over the swath and is below $-30 \mathrm{~dB}$. The range ambiguity suppression is mainly achieved by the narrow SCORE receive beam, rather than by the transmit beam (see also Figure 7(b) where the first range ambiguity is within the main lobe of the transmit beam). In fact, the useful echo is always, that is, for each point on the swath, weighted by the maximum of the receive pattern, whereas the ambiguous echoes are strongly attenuated by the receive pattern sidelobes. The total ambiguity-to-signal ratio, that is, the sum of $A A S R$ and $R A S R$, is mainly determined by the $A A S R$ since it is about $10 \mathrm{~dB}$ larger than the RASR.

Figure 9 shows the NESZ versus ground range position along the swath. The NESZ is below $-26 \mathrm{~dB}$ all over the swath. This assessment is mainly related to the transmitted power and to the area of the antenna in transmission and reception. The trend of the curve along the swath is due to the transmit pattern shape. Here, the antenna tilt angle is such that the maximum of the transmit antenna pattern is closer to the far range. This improves the NESZ in far range with respect to the near range, so that the resulting NESZ is symmetric in near and far range.

Figure 10 describes the effect of topographic height on SCORE performance. The dotted curve corresponds to a source with elevation $1500 \mathrm{~m}$. The solid one, reported as a benchmark, corresponds to a source with no elevation. Figure 10(a) shows the displacement between the receive pattern steering angle and the actual direction of arrival, 


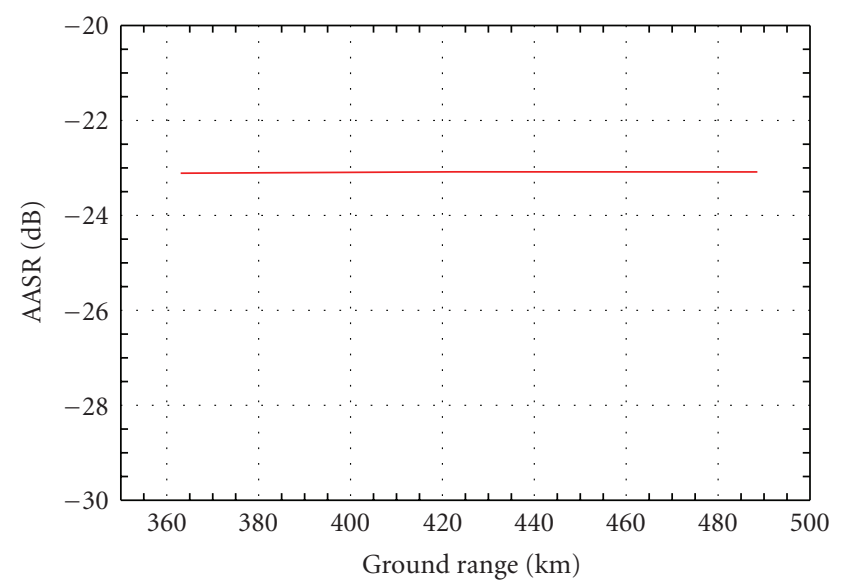

(a) $A A S R$

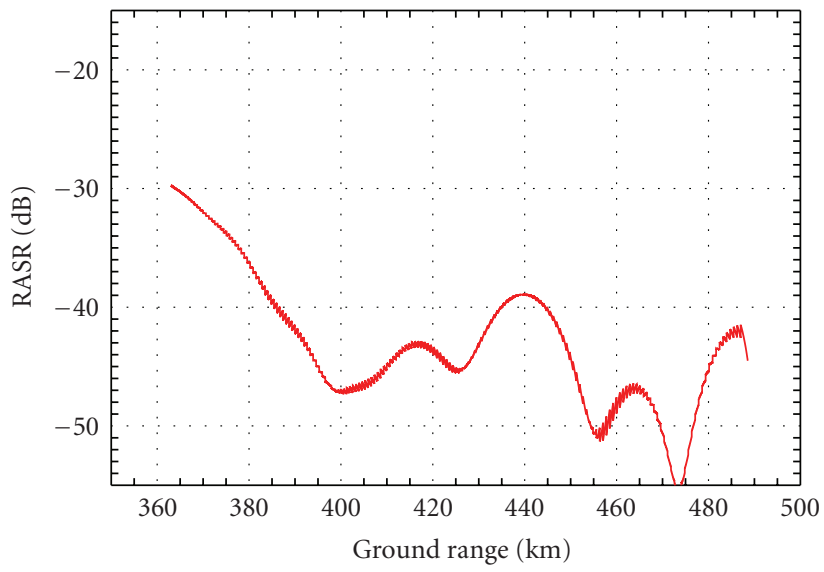

(b) RASR

Figure 8: Ambiguities versus ground range.

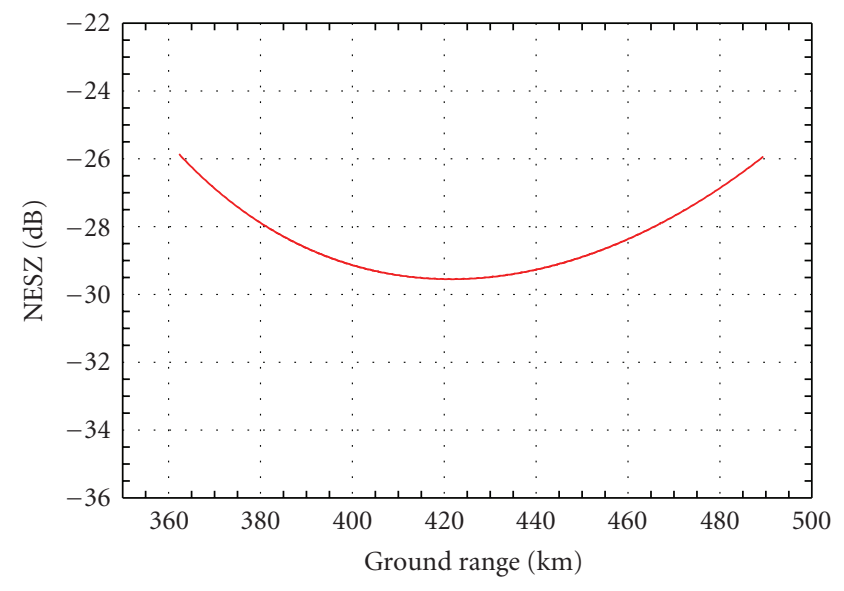

Figure 9: The noise-equivalent sigma zero of the planar system versus ground range.

versus the location of the source along the swath. The steering error is more severe in near range than in far range, due to the spherical geometry of the earth surface. Its value is in the order of $0.2^{\circ}$. The corresponding SCORE pattern loss depends on the beamwidth of the receive pattern: the broader the beamwidth, the more the system is robust to surface elevation. For the current system the loss varies over the range between $-0.15 \mathrm{~dB}$ and $-0.25 \mathrm{~dB}$.

The dashed line in Figure 10 shows that the SPL for a topographic height of $5 \mathrm{~km}$ may get in the order of $-2.5 \mathrm{~dB}$. Although it is not common to have elevation variations of this order within one imaged scene, however different Earth regions may well have height variations of several kilometers. As a result it is concluded that the instrument commanding, that is, beam steering, should take the scene height into account, however an adaptation of the beam steering within one data take is not required.

The pulse extension loss of the planar system, shown in Figure 11, is below $0.4 \mathrm{~dB}$ throughout the swath. This is due to the low duty cycle of $8 \%$ which results in a pulse extension much smaller than the beamwidth of the elevation pattern. To be able to operate SCORE with a low PEL even with long chirp pulses $[6,7]$ suggests an additional frequency dependent beam steering. This can be understood as an intentional frequency dispersion which exploits the linear frequency variation within a pulse (chirp). Effectively, different beams are generated for different frequencies which correspond to different steering directions. This technique, which is implemented through modification of the SCORE steering coefficients, effectively removes the PEL.

\section{Design and Performance of Reflector System}

In analogy to the previous section, a design example, based on the reflector antenna principle, with a feed array shall be demonstrated.

7.1. Reflector System Design. As stated in Section 5, the design goal is to achieve similar half power beam widths in elevation and azimuth as in the planar array case, in order to meet the performance requirements presented in Table 1 . The degrees of freedom for the conceptual design are the focal length, the diameter, and the feed array geometry [16].

The schematic design of the reflector antenna with feed array is presented in Figure 12. The feed array is positioned symmetrically in front of the reflector in the focal plane $z=F$, neglecting the effect of blockage. Here the elevation plane is defined in the Cartesian reflector coordinate system with $y=0$, with the azimuth direction orthogonal to this plane. The diameter $d$ of the reflector is $10 \mathrm{~m}$. With a focal length $F$ of $5.7 \mathrm{~m}$, the shape parameter $a$, which defines the $z$-coordinate of each surface point of the paraboloid, can be determined as $a=1 / 4 F$.

The feed array is a linear array consisting of 26 elements, facing the plane $z=F$. This allows beam steering in elevation direction. The spacing $\Delta x_{f}$ of adjacent elements is $0.58 \lambda$. For quadratic $0.4 \lambda$ patches, the dimensions of the (passive) patch array, allowing for sufficient margin, is approximately $85 \mathrm{~cm} \times 5 \mathrm{~cm}$. The array design is not 


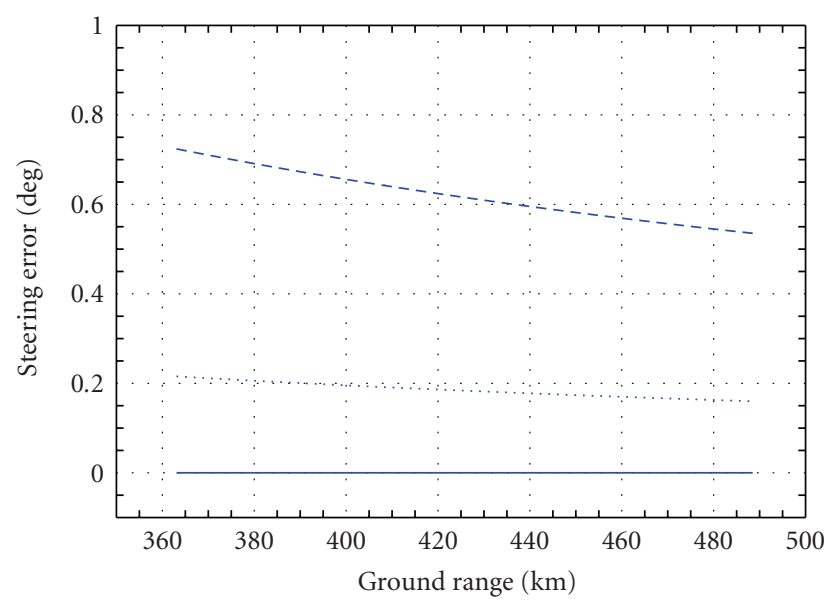

(a) Steering error

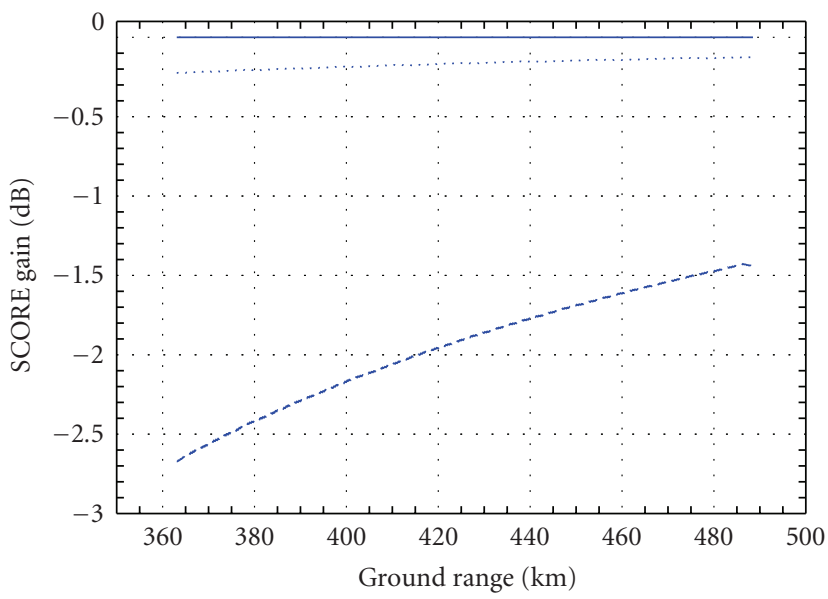

(b) $S P L$

FIGURE 10: SCORE performance versus ground range for $0 \mathrm{~m}$ (solid line), $1500 \mathrm{~m}$ (dotted line), and $5 \mathrm{~km}$ (dashed line) topographic height, respectively.

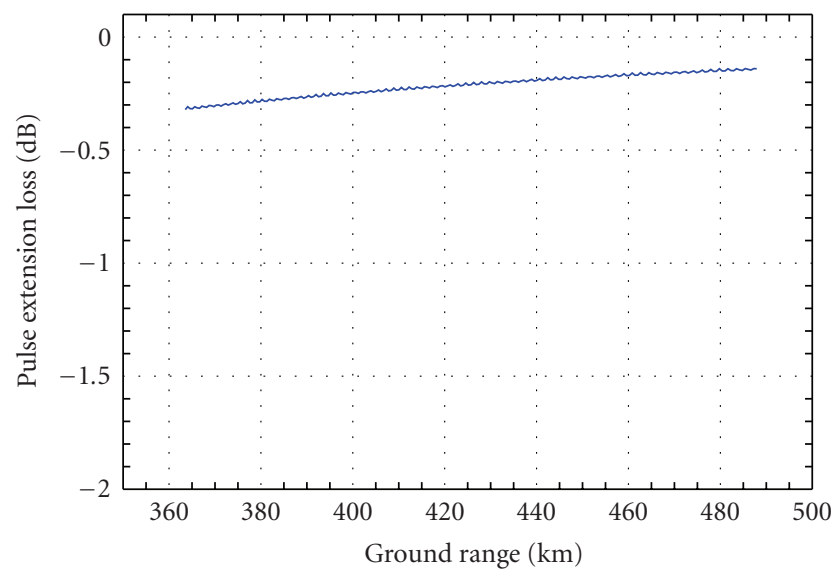

Figure 11: The pulse extension loss of the planar system versus ground range.

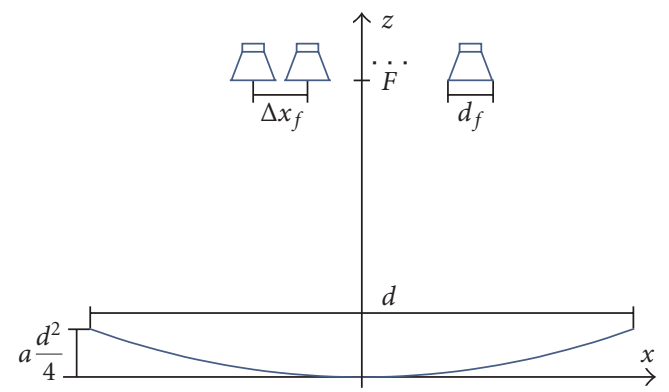

Figure 12: C-Band reflector and feed array geometry.

required to allow for the generation of multiple azimuth beams (cf. discussion in Section 2.2), since a single azimuth beam provides sufficient Doppler bandwidth to achieve the required azimuth resolution of $8 \mathrm{~m}$ (see Table 1).
A simplified Geometrical Optics approach was used for the pattern and field computations of the system under consideration. The results were verified with reference data obtained from commercial software (GRASP9), showing a maximum relative error $\leq 1.5 \%$ within the angular range of the 5th order sidelobe.

SCORE Feed Selection. The beam-forming capabilities of a reflector antenna are more restricted compared to a planar array antenna, since the subpatterns do not overlap substantially. Therefore the pattern is generated by turning feed elements on or off. As the pulse travels over ground, the field distribution in the focal plane changes and other elements have to be switched on. The feed selection has a significant impact on the system performance in terms of NESZ and RASR. The goal of the feed selection strategy is to optimize these parameters.

In general, the optimum selection is the one which results in the maximum antenna gain or equivalently the highest illumination efficiency. As indicated by the dotted curves in Figure 13, the highest gain is achieved with two activated feed elements. A single active element will increase the spill over and by this decrease the illumination efficiency (gain), whereas three or more activated elements will reduce both spill over and aperture efficiency, which also results in a lower gain. Nevertheless, two active receive elements would result in a high pulse extension loss. Consequently, the minimum number of active elements has to be increased for a wider beamwidth. In case of the system given here, four active elements are required, at the cost of a reduced gain. Note that this is a particularity of the system architecture considered here for the reflector system. Other solutions, overcoming these limitations, are discussed in Section 8.

The field strength distribution on the feed plane due to an extended pulse is also indicated in Figure 14, where the field is concentrated over a subset of feed elements. The activated elements are represented by black patches, and 


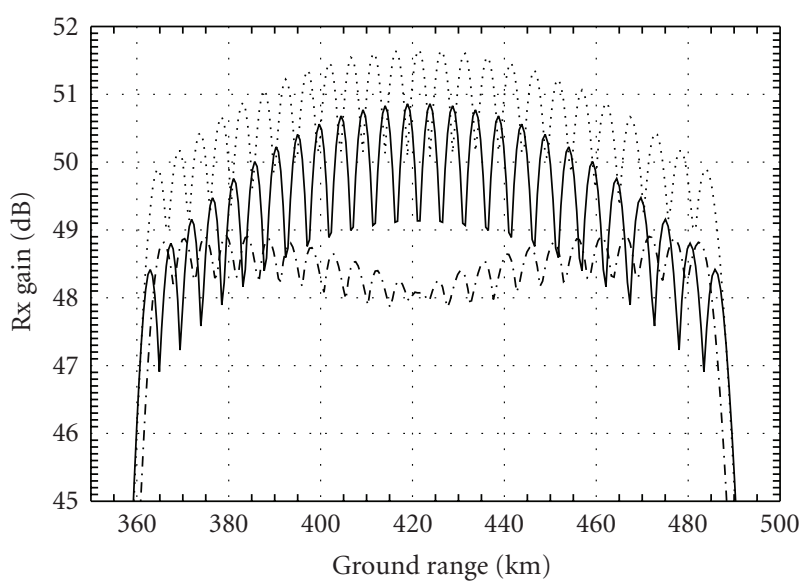

FIGURE 13: Reflector gain pattern versus ground range with one (solid), two (dotted) and four (dashed) feed elements activated.

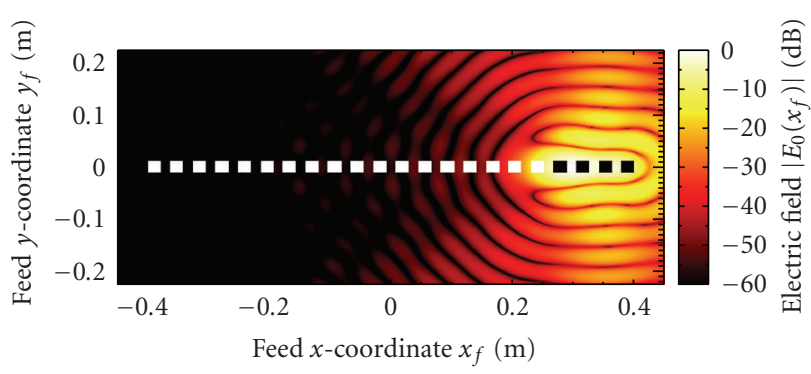

FIGURE 14: Normalized electric field on the feed array due to an extended pulse on the swath edge.

clearly activating only two elements would result in a power loss.

To get an impression of the pattern shapes, the transmit pattern and one receive pattern have been computed. Figure 15 shows a polar plot of the transmit pattern, $C_{T}$, weighted with the maximum gain. Here all 26 feed elements are activated, generating a narrow beam in azimuth and a broad homogeneous beam in elevation, with a half power beamwidth $\Theta_{T, \mathrm{el}}=7.3^{\circ}$.

In the receive case an example of the antenna pattern is presented in Figure 16, where the same four off-center feed elements are activated as in Figure 14. Due to the reciprocity principle, the pattern has basically the same shape as the field distribution. The half power beamwidth in elevation, $\Theta_{R, \mathrm{el}}$, is in the order of $1.2^{\circ}$, covering the complete pulse on ground.

Figure 17(a) shows a cut in the azimuth plane. The solid lines represent the transmit patterns (all elements turned on), while the dashed curves indicate the receive patterns when only four center elements are turned on. All patterns are normalized to the maximum gain which would be achieved for an optimum aperture efficiency (note that this maximum gain is not reached for the system considered here). Both plots show the high directivity of the receive pattern with respect to the transmit pattern; the difference is approximately $8.6 \mathrm{~dB}$. In azimuth, Figure 17(a), only a single beam is generated; however this beam slightly depends on

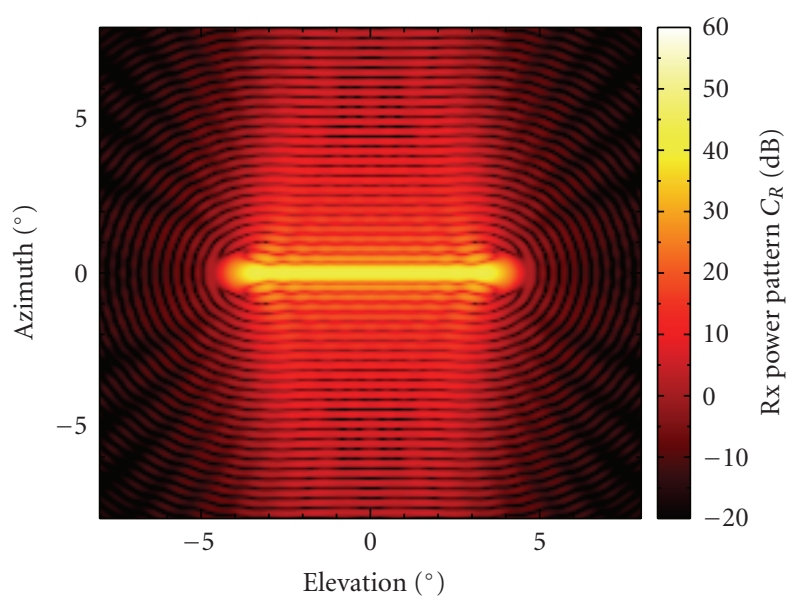

Figure 15: Transmit power pattern with 26 elements activated.

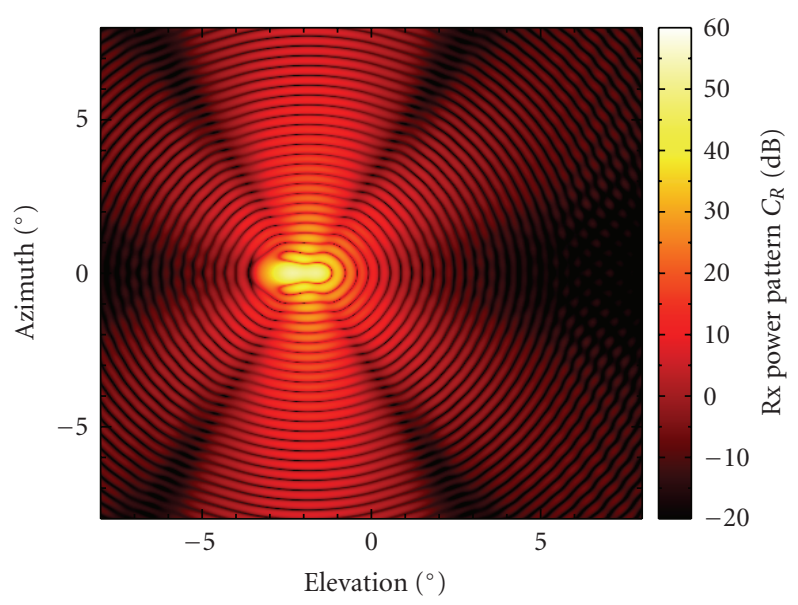

FIGURE 16: Receive power pattern with 5 elements activated.

the number of active elevation elements as can be seen by the slight shift in position of the first nulls. The plot for the elevation plane, depicted in Figure 17(b), shows a nearly rectangular shaped transmit pattern, a property inherent to reflector antennas. This results in an efficient concentration of the transmitted energy to the ground region of interest and manifests itself through an improved NESZ performance.

7.2. Reflector System Performance. The ambiguity performance in terms of AASR and RASR is shown in Figure 18. The processed Doppler bandwidth, $B_{p}$, has been adapted to achieve the same $A A S R$ of $\approx-22 \mathrm{~dB}$ as in the planar array system. The processed Doppler bandwidth is indicated by the center bar at the top of Figure 17(a); further the ambiguous spectral domains, marked with the off-center bars, coincide well with the first pattern nulls. The processed Doppler bandwidth is $1150 \mathrm{~Hz}$, yielding an azimuth resolution, $\delta_{\mathrm{az}}$, of approximately $6 \mathrm{~m}$.

For the range-ambiguity-to-signal ratio the low $P R F$ has the opposite effect as for the azimuth ambiguities. Principally a lower $P R F$ will improve the RASR. The angular 


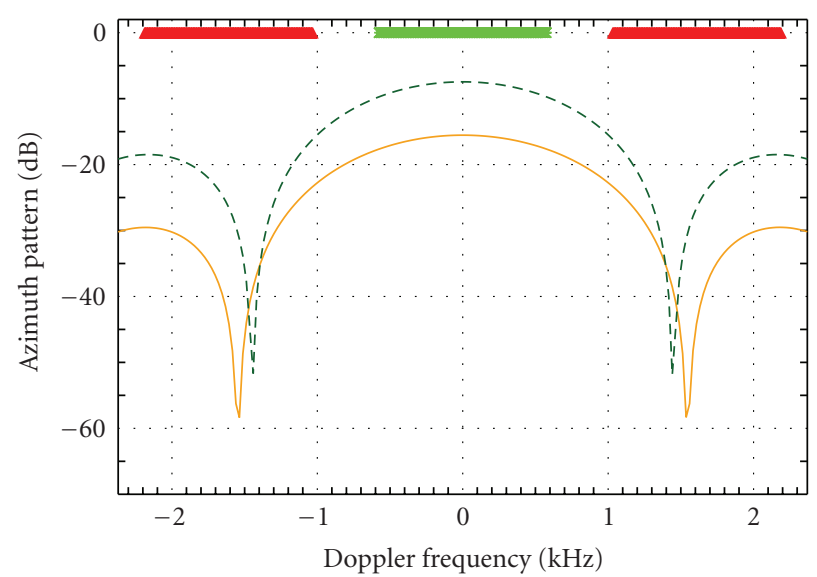

(a) Azimuth patterns

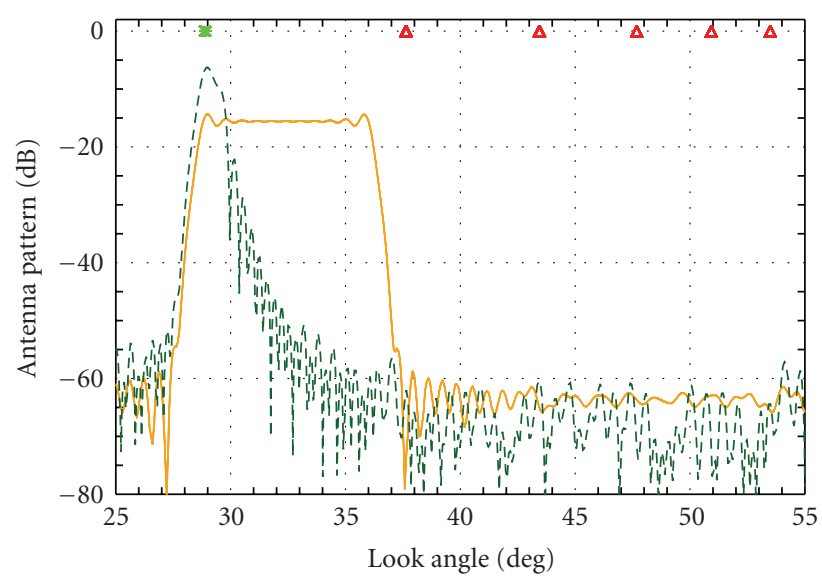

(b) Elevation patterns

FIGURE 17: Transmit (solid line) and receive patterns (dashed line) of the reflector antenna system. All patterns are normalized to the gain achieved for a optimum aperture efficiency.

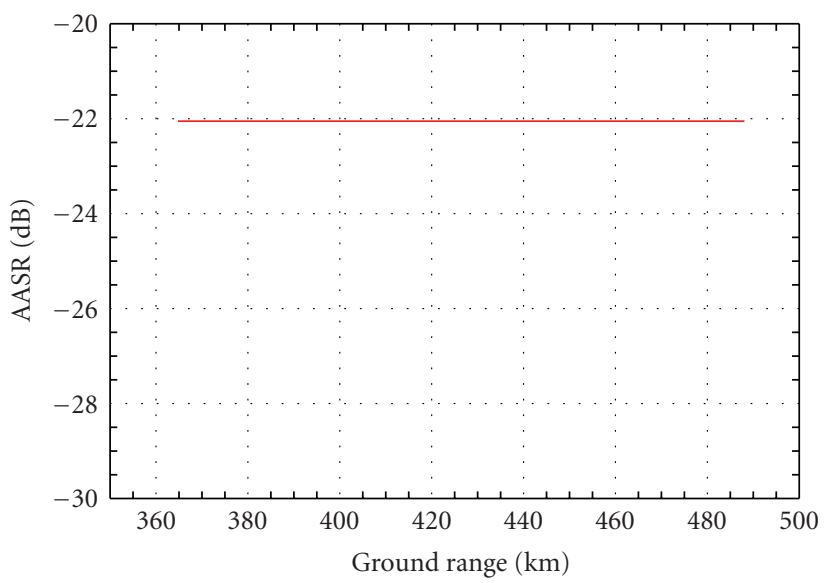

(a) $A A S R$

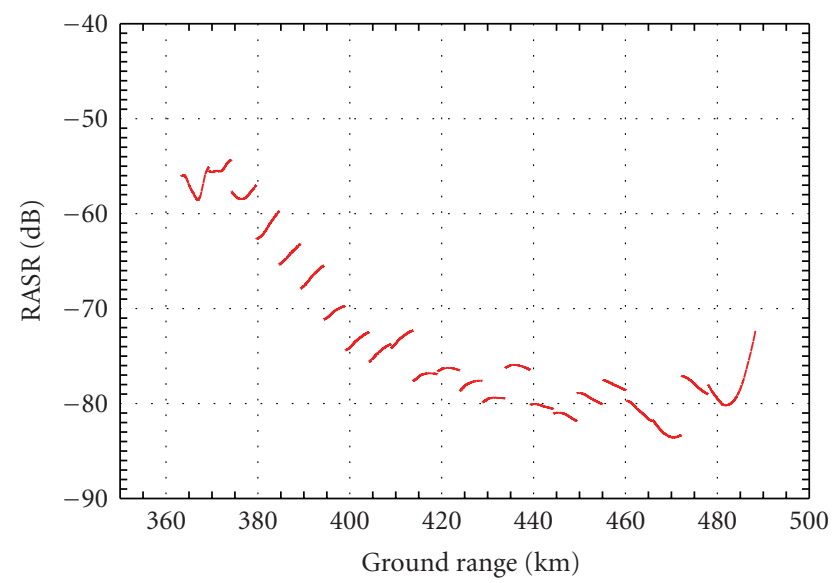

(b) RASR

Figure 18: Ambiguities versus ground range for the reflector system.

position of the first range ambiguity is outside the mainlobe of the transmit and receive patterns, as can be seen in Figure 17(b). This results in the good range-ambiguitysuppression in Figure 18(b) of better than $-50 \mathrm{~dB}$. For a real reflector the realistic suppression will be less and dictated by imperfections which deteriorate the ideal patter.

The NESZ over ground range is presented in Figure 19. The two curves are computed for the cases that either two or four successive elements are activated. The loss in NESZ in the four element case (upper curve) results from the reduced receive gain. The notches in the curve shape mark the switching of the active elements and the corresponding pattern switching.

The NESZ loss at the swath borders can be compensated for with a slightly increased feed array, however, due to the rectangular transmit pattern shape, the NESZ will be worse in the far range of the swath (an adaption of the tilt angle to compensate, as done for the planar system, is not possible here). The reason for showing the NESZ of two

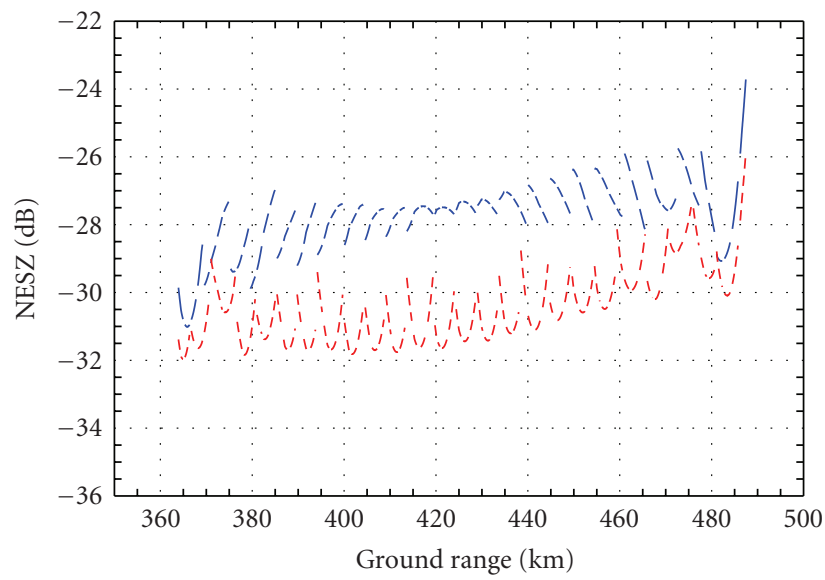

Figure 19: The noise-equivalent sigma zero of the reflector system versus ground range for two (solid curve) and four (dashed curve) active feed elements. 


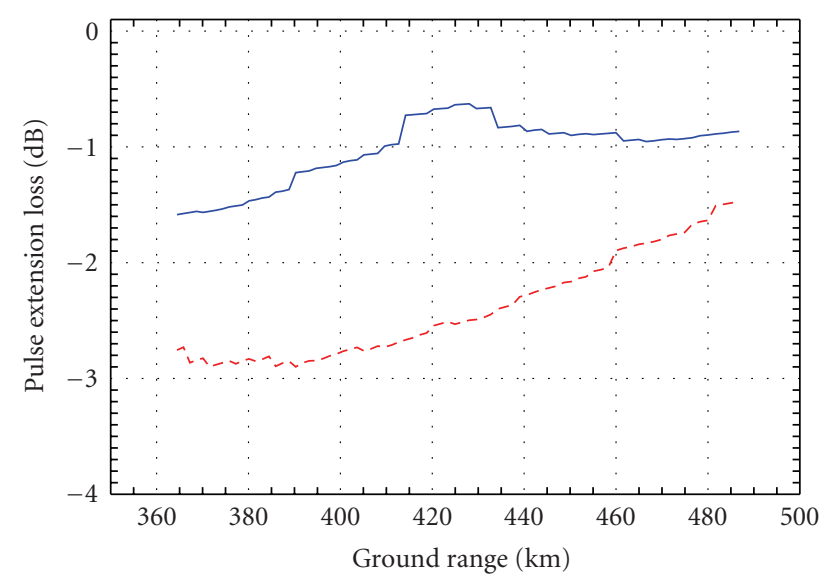

FIgure 20: The pulse extension loss of the reflector system versus ground range with four (solid line) and two (dotted line) active elements.

active elements (lower curve), is that a more sophisticated digital signal processing could enable this improve in NESZ by compensating the reduced beamwidth (see Section 8).

The pulse extension loss of the reflector system (cf. Figure 20 solid line) is higher than for the planar system and reaches values up to $1.7 \mathrm{~dB}$ at near range. Here, the PEL is an additional loss also effecting the value of the NESZ, that is, the curves in Figure 19 are shifted by an amount corresponding to the PEL. Increasingly unsymmetrical patterns for off-center beams cause an increased loss (see definition of $P E L$ in Section 4). For comparison the PEL for two activated elements is also included in the plot (dashed line) indicating the higher loss of up to $3 \mathrm{~dB}$ in this case.

\section{Conclusion}

This paper compares two innovative antenna architectures that employ the technique of digital beam-forming on receive to improve the imaging performance and radiometric resolution of future SAR systems without loosing wide swath coverage. Both architectures rely on the side-looking geometry of a spaceborne imaging radar where the scattered echoes arrive at each instance of time only from a very narrow range of angles. A real time angular steering of a high-gain receiver beam is hence well suited to collect all scattered radar echoes from a wide swath without any information loss. The originally suggested architecture for such a digital beam-forming radar employed a planar antenna array [7]. The beam steering in this architecture requires the combination of all recorded antenna signals in a complex real time processor. This puts a high demand on the digital hardware, especially if one seeks for a high receiver gain by using an extended Rx array with a large number of antenna elements.

As an alternative to a planar antenna, we have introduced the novel idea to combine a reflector antenna with a digital feed array to enhance the performance of future SAR systems. Large unfoldable reflectors are now a mature technology with extensive flight heritage in space telecommunications and lightweight mesh reflectors with diameters of $20 \mathrm{~m}$ and more will be deployed in space in the near future. Unfoldable reflector antennas have therefore a high potential to significantly increase the receiving aperture and by this both the range ambiguity suppression and the NESZ if compared to planar arrays where the size of the antenna is usually restricted by the limited space in the launcher fairing. This is indicated in the computed NESZ values for the two systems (compare Figures 9 and 19) which show an NESZ improvement for the reflector system when operated with two active receive elements.

The reflector concentrates at each instant of time the signal power of the received wave fronts from the swath echoes onto a small number of feed elements. In consequence, only a small subset of all feed element signals has to be combined in real time, thereby reducing the complexity of the beamforming processor also for large apertures. A very simple implementation of a "digital beamformer" is provided by a mere switching between individual feed elements. The element selection can be made signal adaptive, for example, by choosing only those feed elements that exceed a given threshold or by selecting the feed element(s) that receive(s) maximum signal power. This fully automatized feed selection eases the instrument commanding and avoids moreover the beam steering loss from topographic height induced mispointings.

Long chirp signals and large reflectors may, however, require more sophisticated signal processing techniques to optimize the performance. In fact, in this case, the achievable beamwidth is smaller than the instantaneous ground scattering field induced by a long chirp signal. The straight-forward solution for long chirp signals could be a simple increase in the number of selected feed elements and a subsequent summation of their output signals, but this will inevitably reduce the antenna gain, thereby making only suboptimum use of the high Rx-gain capabilities of a large reflector. Such a gain loss from long chirps can be avoided by taking into account that each feed element receives at each instant of time only a fraction of the scattered radar pulse and hence only a corresponding portion of the transmitted frequency spectrum. It is therefore possible to split the incoming signals from the feed elements into multiple frequency subbands and to select for each sub-band again only the element(s) with maximum signal power. This trick can be regarded as providing a dispersive Rx-beam that ensures a high antenna gain for every frequency component of a long chirp. In consequence, one can take full advantage of the large apertures provided by present and future deployable reflector antennas. Reflectors with digital feed arrays are therefore a promising concept for future SAR systems with high potential to outperform planar arrays with regard to the SAR imaging performance for a given weight, size and cost budget.

\section{Acknowledgment}

The authors greatly appreciate the detailed comments and suggestions of the anonymous reviewers, which pushed them to an in-depth treatment of the subject leading to a significantly improved paper. 


\section{References}

[1] G. Krieger, N. Gebert, and A. Moreira, "Multidimensional waveform encoding: a new digital beamforming technique for synthetic aperture radar remote sensing," IEEE Transactions on Geoscience and Remote Sensing, vol. 46, no. 1, pp. 31-46, 2008.

[2] G. Krieger, N. Gebert, M. Younis, F. Bordoni, A. Patyuchenko, and A. Moreira, "Advanced concepts for ultra-wide swath SAR imaging," in Proceedings of the 7th European Conference on Synthetic Aperture Radar (EUSAR '08), Friedrichshafen, Germany, June 2008.

[3] C. Fischer, C. Schaefer, and C. Heer, "Technology development for the HRWS (High Resolution Wide Swath) SAR," in Proceedings of International Radar Symposium (IRS '07), Cologne, Germany, September 2007.

[4] J. H. Blythe, "Radar systems," U.S. Patent 4253 098, February, 1981.

[5] J. T. Kare, "Moving receive beam method and apparatus for synthetic aperture radar," U.S. Patent 6175 326, January 2001.

[6] M. Suess and W. Wiesbeck, "Side-looking synthetic aperture radar system," European Patent EP 1241 487, September 2002.

[7] M. Suess, B. Grafmueller, and R. Zahn, "A novel high resolution, wide swath SAR system," in Proceedings of International Geoscience and Remote Sensing Symposium (IGARSS '01), vol. 3, pp. 1013-1015, Sydney, Australia, July 2001.

[8] M. Younis, C. Fischer, and W. Wiesbeck, "Digital beamforming in SAR systems," IEEE Transactions on Geoscience and Remote Sensing, vol. 41, no. 7, part 2, pp. 1735-1739, 2003.

[9] G. Krieger, N. Gebert, and A. Moreira, "Unambiguous SAR signal reconstruction from non-uniform displaced phase centre sampling," IEEE Geoscience and Remote Sensing Letters, vol. 1, no. 4, pp. 260-264, 2004.

[10] A. Papoulis, "Generalized sampling expansion," IEEE Transactions on Circuits and Systems, vol. 24, no. 11, pp. 652-654, 1977.

[11] J. L. Brown Jr., "Multi-channel sampling of low-pass signals," IEEE Transactions on Circuits and Systems, vol. 28, no. 2, pp. 101-106, 1981.

[12] J. C. Curlander and R. N. McDonough, Synthetic Aperture Radar Systems and Signal Processing, John Wiley \& Sons, New York, NY, USA, 1991.

[13] M. Younis, "General formulation of NESZ," Technical Note TN-SAR-Tech-001, German Aerospace Center, Microwaves and Radar Institute, Wessling, Germany, March 2008.

[14] S. Mezzasoma, A. Gallon, F. Impagnatiello, et al., "COSMOSkyMed system commissioning: end-to-end system performance verification," in Proceedings of IEEE Conference on Radar (RADAR '08), pp. 1092-1096, Rome, Italy, May 2008.

[15] TerraSAR-X, German Aerospace Center (DLR), September 2008, http://www.dlr.de/TerraSAR-X/.

[16] C. Balanis, Antenna Theory: Analysis and Design, John Wiley \& Sons, New York, NY, USA, 1997. 

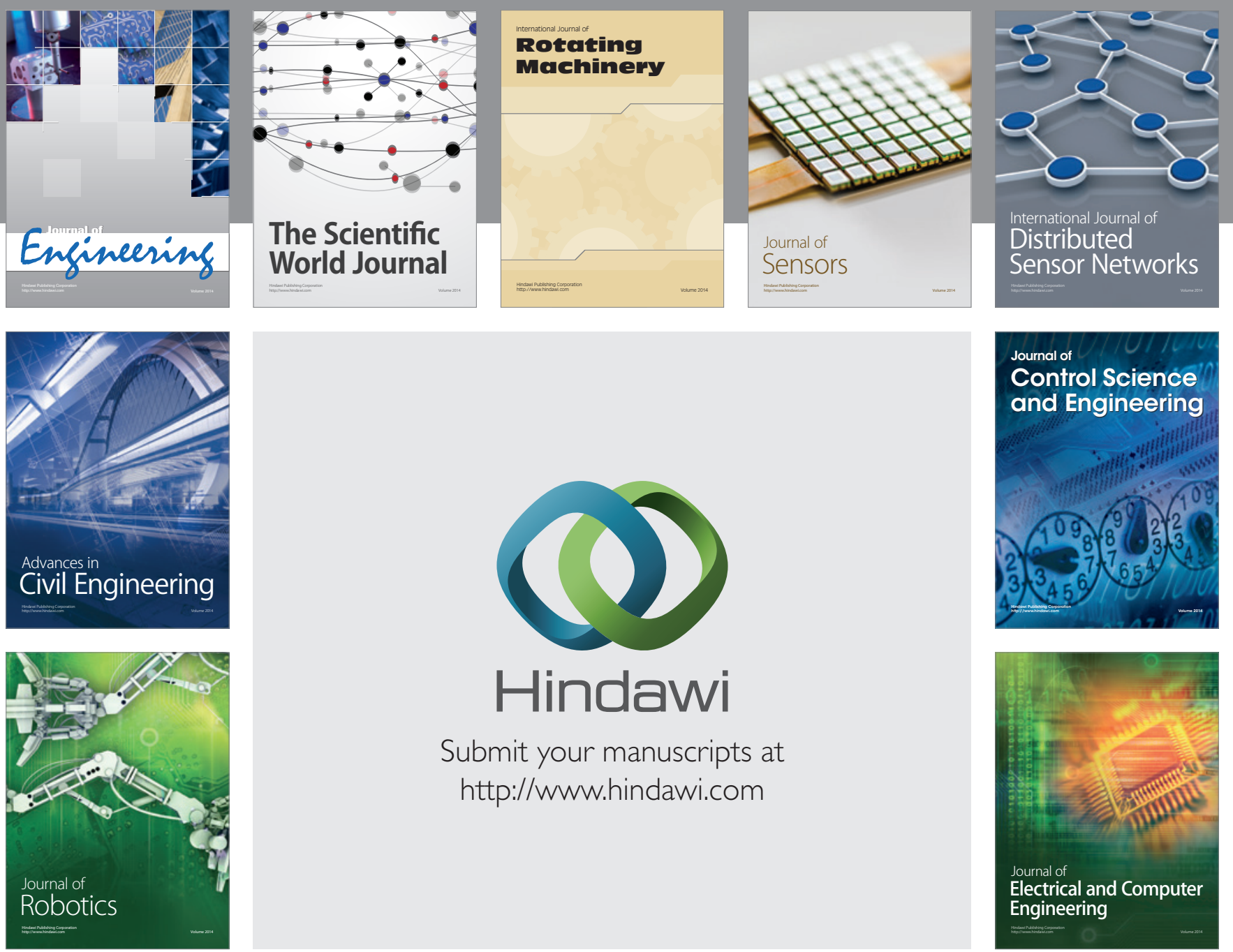

Submit your manuscripts at

http://www.hindawi.com
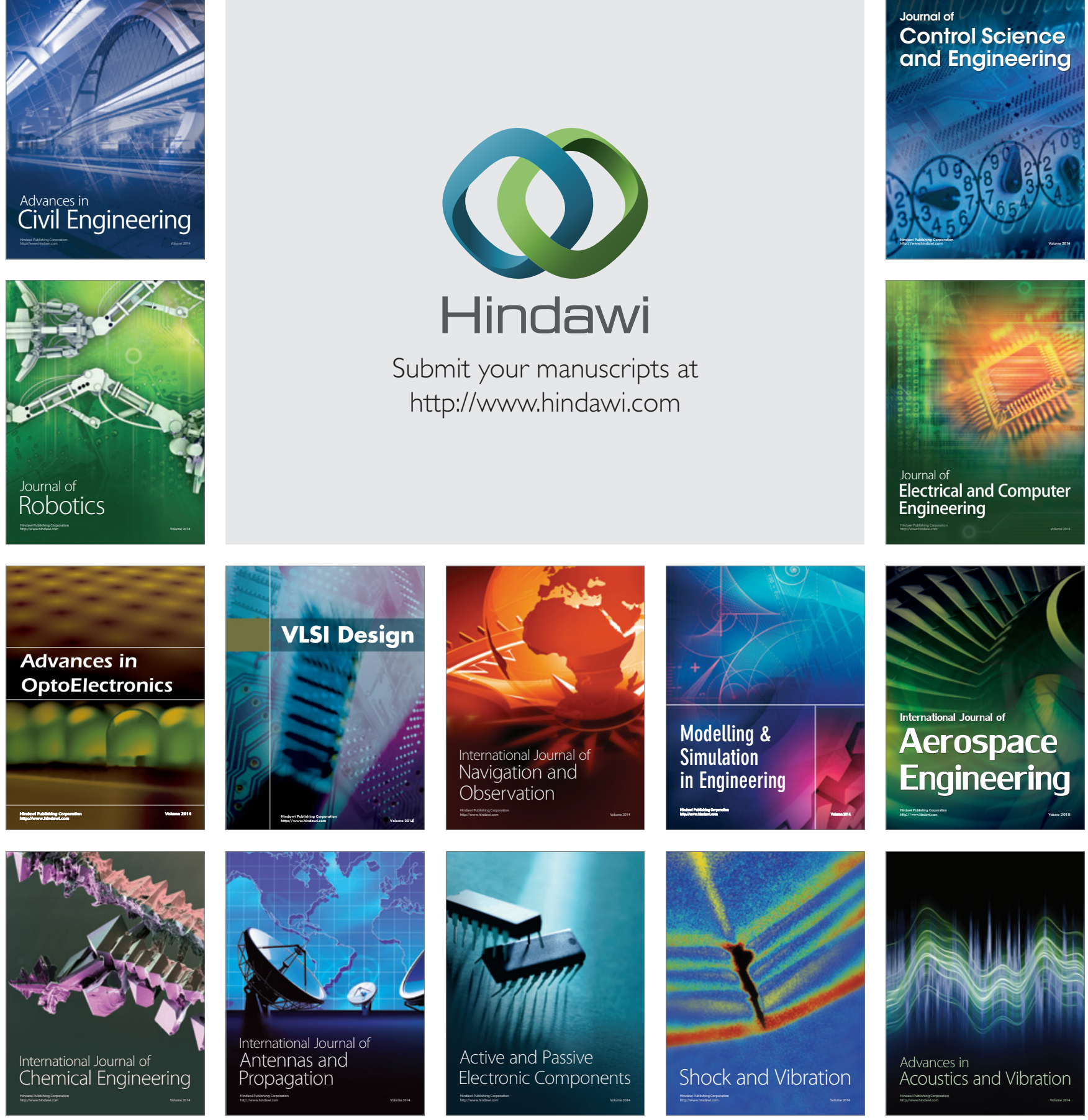